\title{
Electrical Measurements on Musa Acuminata Colla
}

\author{
J. Omprakash, N. V. Prasad ", G. Prasad, V.G. Krishnan, G. S. Kumar \\ Materials Research Laboratory, Department of Physics, Osmania University, Hyderabad 50007, India \\ *Corresponding Author: nvp_phy@osmania.ac.in
}

Copyright (C 2014 Horizon Research Publishing All rights reserved.

\begin{abstract}
Typical Electrical feature of Musa acuminata colla (common name of commercially available banana) peel was identified as inhomogeneous lossy-dielectric. Considering in this direction, detailed impedance analysis was made at room temperature in the frequency range of $0.001 \mathrm{~Hz}$ to $1 \mathrm{M} \mathrm{Hz}$. Impedance relaxation was well-fitted into Gaussian curves. The complex impedance plot, associated with two depressed semicircles, indicates Warburg-diffusion phenomenon. Randles's plot and frequency vs. admittance plot confirm the same. The results were corroborated by Jonscher's Universal law for better understanding of the inhibitor (corrosion-free) nature of the banana-peel.
\end{abstract}

Keywords Impedance, Dielectric Complex Impedance Plots, AC-conductivity, Randles Plot, Warburg-diffusion

\section{Introduction}

Archaeological evidence suggests that banana cultivation was started during 8000 to 5000 B.C. in the western highlands of Province of Papua New Guinea. The common name of the banana is known as Musa acuminata colla. In 6000 B.C. the word banana was mentioned in Buddhist textbook and it should be highlighted here that Alexander, the grate, discovered the taste of banana in the Indian valley in 327 B.C. In addition to this, many religious books quoted banana, and it is considered to be a sacred-fruit (god's gift).

The characteristic feature of ferroelectric material is to show a peak in the dielectric-temperature data as well as a hysteresis loop in the polarization vs. electric field data. It is pointed out that many lossy-dielectric materials, exhibits a kind of nonlinear loops due to surface-polarization phenomenon and these loops were named as artefact-hysteresis loop (often referred as banana loops) [1-4]. The characteristic occurrence of the banana - loop is considered to be a hallmark for the lossy-dielectric material [5], which has no relationship to the ferroelectric nature (see Yago et al and references) [5]. This artifact becomes problematic in the field of multi ferroics/magnetoelectrics, where both the magnetic as well as ferroelectric ordering exists. The artefact hysteresis loops for multifarious were attributed to the magnetic ions or oxygen vacancies or a combination of both [6]. In order to interpret the loss-dielectric data a black-box approach is needed.

Loidl et al [7] demonstrated the paraelectric behaviour of banana peel (sample), using different contacts, on different geometrical size samples by measuring hysteresis loop as a function of frequency. In addition to this, the dielectric spectrum has shown a sequence of relaxations. This has prompted us to have a relook at the same phenomenon through impedance analysis.

Impedance spectroscopic measurement is considered to be a powerful technique and it has been widely used to characterize the dielectric behaviour of many ordered/disordered materials. The microscopic origin of impedance spectroscopy is to understand the localized defects in a closed proximity. Based on the present literature survey, the global changes of inhomogeneous dielectric materials, introduced by defects, can be studied by means of impedance spectroscopic studies. The main interest of this work is to study the intensified relaxations, observed in the conductivity as well as impedance spectroscopic plots. Since room temperature impedance spectroscopic plots give limited information, the complex, modulus and conductivity data was extracted from the impedance data for further understanding of relaxation behaviour.

\section{Experimental}

Impedance measurements were made, using Solitron low frequency impedance Analyzer in the frequency range of $1 \mathrm{~m}$ $\mathrm{Hz}$ to $1 \mathrm{MHz}$ at room temperature. In the present investigation, we used commercially available banana peel as a sample. It is probably true to say that the influence of electrode-effects is not yet clearly understood. In order to ensure reproducibility in the impedance data, different sample-holders $(\mathrm{Cu}$ and $\mathrm{Au})$ are used in the present investigation. Separately designed two-homemade sample-holders are shown in fig $1 \mathrm{a}$ and fig $1 \mathrm{~b}$ respectively. Fig la shows gold-sputtered electrode sample holder (in air medium) and fig $1 \mathrm{~b}$ shows copper-electrode sample holder (immersed in a silicon oil bath). Here $\mathrm{Cu}$-electrode sample holder is used for stabilizing the low temperature data (below room temperature). 
Impedance measurements carried out within relaxation frequency may give ambiguous results, when compared with those obtained at arbitrary selected frequencies. In this connection impedance measurement carried out in frequency scan, which enable us to eliminate errors like stray frequency effects. The impedance measurements on a material give us data having both resistive impedance (real part of impedance Z') and reactive impedance (imaginary part of impedance Z") components. The impedance data of both spectroscopic as well as complex (Cole-Cole) plot is used to understand the dielectric relaxation of the sample. In principle, complex impedance plots are used to deduce the possible equivalent circuit. In some aspect, these plots may not give us sufficient information, therefore, same impedance data is re-plotted on logarithmic scale or transformed into another formalism like admittance $\left(\mathrm{Y}^{*}\right)$, permittivity $\left(\varepsilon^{*}\right)$ and relative electric modulus $\left(\mathrm{M}^{*}\right)$. The impedance formalism $[8,9]$ used in the present investigation is.

$$
\begin{gathered}
Z^{*}=Z^{\prime}-i Z^{\prime \prime}=R_{\mathrm{S}}-1 / \omega C_{\mathrm{S}} \\
\mathrm{Y}^{*}=\mathrm{Y}^{\prime}+\mathrm{i} Y^{\prime \prime}=1 / \mathrm{R}_{\mathrm{P}}+\mathrm{j} \omega \mathrm{C}_{\mathrm{P}} \\
\varepsilon^{*}=\varepsilon^{\prime}-\mathrm{j} \varepsilon^{\prime \prime} \\
\mathrm{M}^{*}=1 / \varepsilon=j \omega \mathrm{C}_{\mathrm{o}} \mathrm{Z}^{*}
\end{gathered}
$$

Where $R_{S}, C_{S}$ are the series resistance and capacitance, $\mathrm{R}_{\mathrm{P}} \mathrm{C}_{\mathrm{P}}$ are the parallel resistance and capacitance respectively, and $\mathrm{C}_{\mathrm{o}}$ is the free space capacitance of the sample holder and electrode arrangement. The real part of capacitance and $\tan \delta$ (loss) of a material can be understood thoroughly by using the following relations.

$\operatorname{Tan} \delta=\varepsilon^{\prime \prime} / \varepsilon^{\prime}=\mathrm{M}^{\prime \prime} / \mathrm{M}^{\prime}=\mathrm{C}^{\prime \prime} / \mathrm{C}^{\prime}=\mathrm{Z}^{\prime} / \mathrm{Z}^{\prime \prime}=\mathrm{Y}^{\prime} / \mathrm{Y}^{\prime \prime}$

\section{Results and Discussion}

Fig 2 shows the variation of the imaginary part of impedance (Z') against frequency on a logarithmic scale. This plot highlights two peaks in the region I \& II, respectively and shows the different features of the response function. The combined real and imaginary part of spectrum specifies the phenomenon of larger resistance and smaller capacitance.

Fig 3 shows the combined plot of both of real and imaginary part of impedance against frequency on the normalized frequency scale ( $\mathrm{f} / \mathrm{fo}$ ). This plot shows symmetry about central frequency and may coincide at higher frequency. The shape and width of the peak are considered to be a characteristic of extended-Debye (or non-Debye) type. This kind of non-Debye confirms the distribution of relations times (more than one relaxation). It should be pointed out here that only a few system shows ideal (Debye) behaviour.

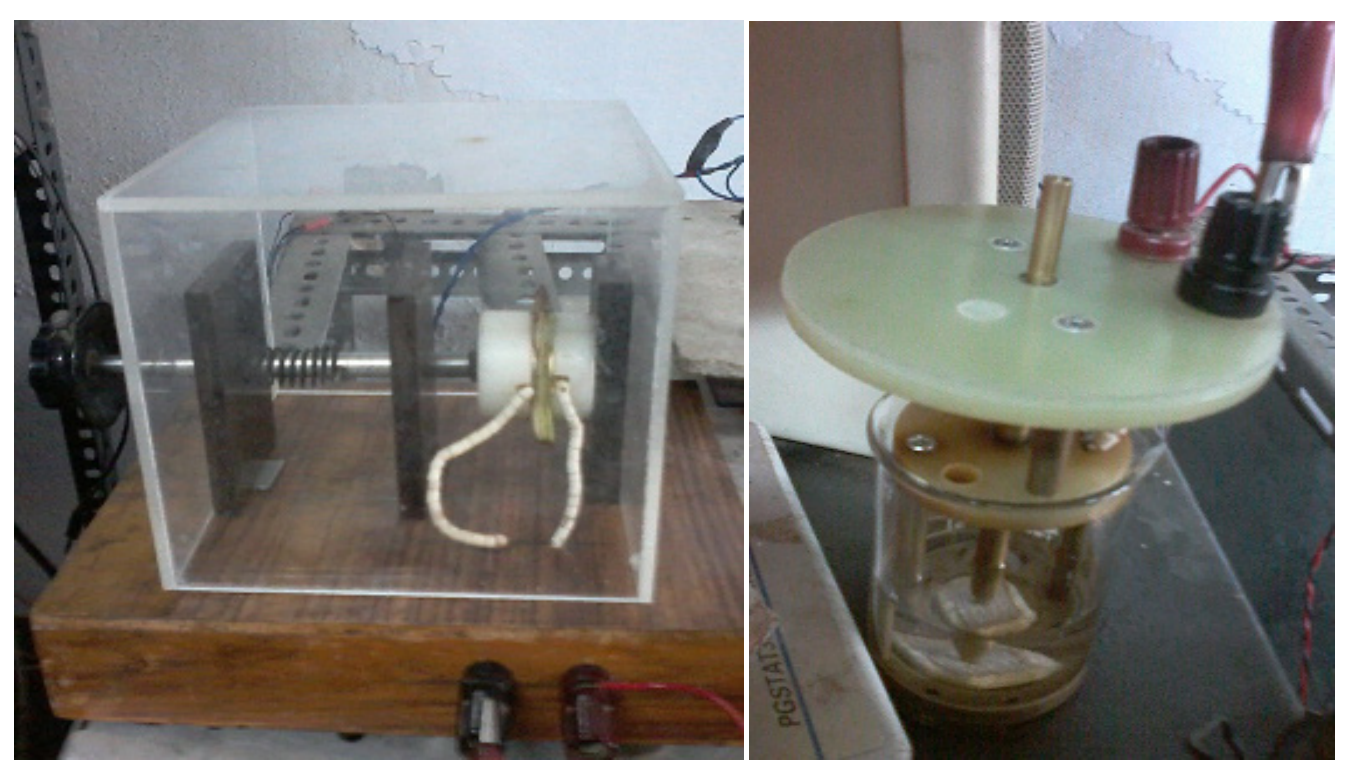

Figure $1 \mathrm{a}$ and $1 \mathrm{~b}$

Figure 1. Sample holder (a) Gold electrodes (in air) (b) Cu-electrodes (in silicon oil bath) 


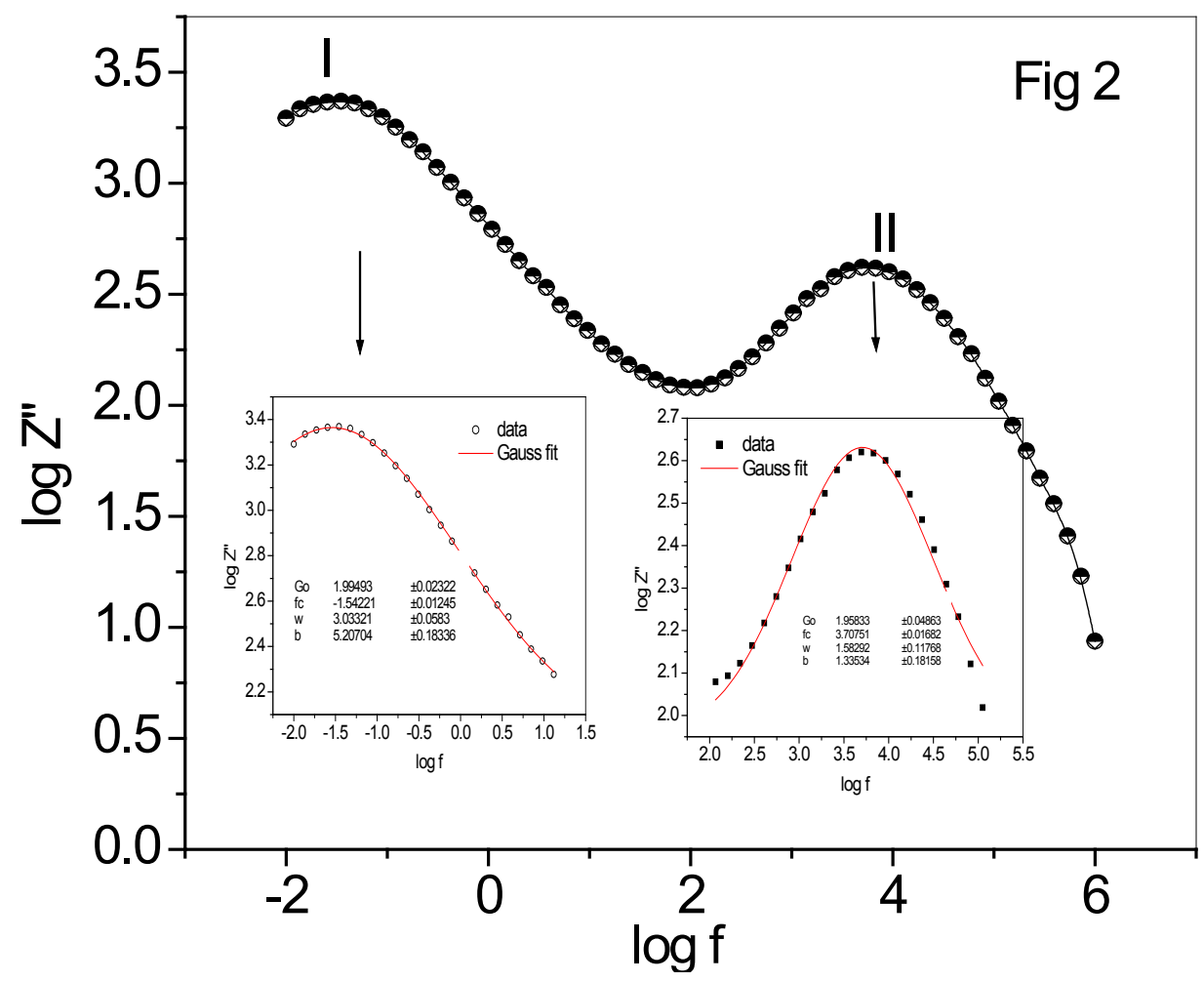

Figure 2. $\log$ Z" vs. $\log f$ (inset fig Gaussian fitting)

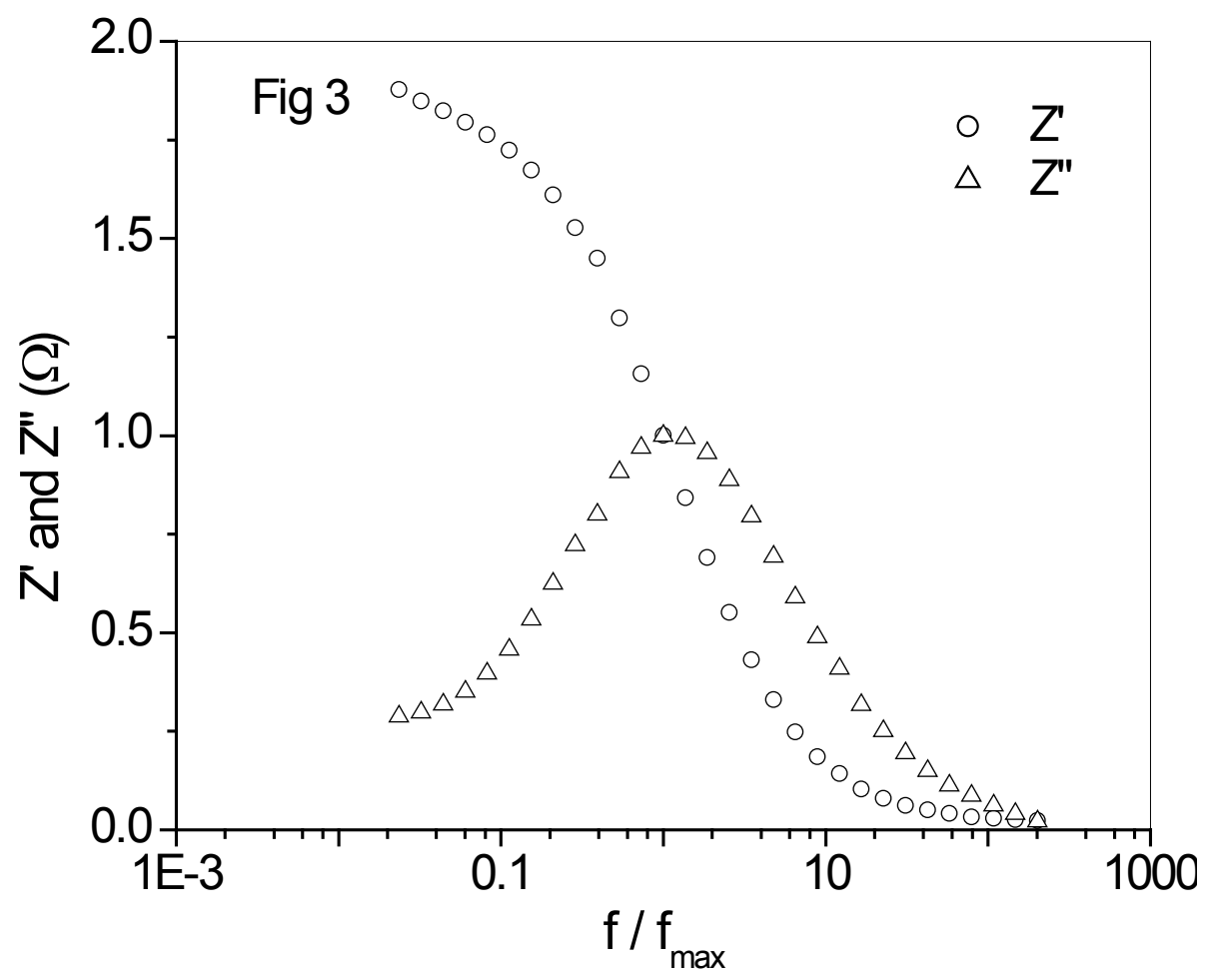

Figure 3. The variation of $Z^{\prime}$ and $Z^{\prime \prime}$ with $\mathrm{f} / \mathrm{f}_{\max }$

Johnson et al [10] reported that the distribution of relaxations was attributed to dipole-dipole interactions followed by the following Gaussian distribution [8]:

$$
G(t)=G o+\frac{b}{w o} \operatorname{Exp}\left[-\frac{2(f-f o)^{2}}{w^{2}}\right]
$$

Here, $G(t)$ is a Gaussian distribution, $b$ is a constant and other terms are its usual significance. In the present investigation, the two peaks observed in the fig 2 , are found to be well fitted with the Gaussian curves, shown as inset fig 2. In addition, the Gaussian peak observed in the region II is found to be considerably broader and the FWHM (full width 
at half maximum) is found to be more than $\log \left(\frac{2+\sqrt{3}}{2-\sqrt{3}}\right)$ Decades. The parameters are mentioned in the plot (inset fig 2).

Complex impedance plots are useful for determining the dominant resistance of the sample and they are insensitive to smaller resistance and therefore complex modulus plots are useful in determining the smallest capacitance. Since the relaxation of different process differs as a consequence of different capacitive compounds, the complex impedance plot is used to represent the response of dielectric material. Based on the distribution of relaxation time and width of the complex impedance semicircles, the common suitable equivalent circuits are depicted in the fig 4 a. The distribution of the relaxation time acts as an origin for CPE (constant phase element). The term CPE was introduced by Jonsher [9] to represent universal capacitor to take into account. The contribution of interacting dipoles is not only due to polarization, but also to the conduction. The generic form of a CPE of impedance can be defined as [8]:

$$
Z^{*}=A\left(j \omega^{\mathrm{n}}\right)^{-1}
$$

Where $\mathrm{A}$ is a constant and $\mathrm{n}$ is related to the depressed-angle of the semicircles. In case of Debye type one should get perfect semicircles, without any depressed angle.

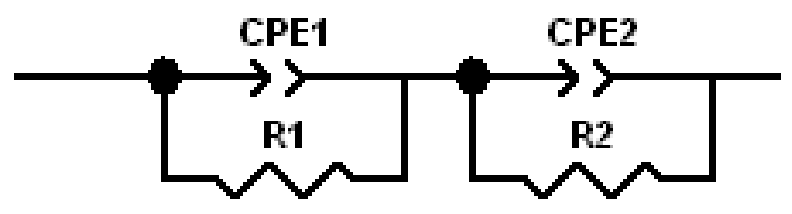

Figure $4 \mathrm{a}$

The banana peel extract contains $[(3 \mathrm{Z}, 7 \mathrm{Z}, 10 \mathrm{Z})$ -1-oxa-6-azacyclododeca-3, 7-10-triene] and the structure of the compound is as shown in fig $4 \mathrm{~b}$. This structure is having excellent mixed type inhibitor efficiency on metal surface [11]. The biological matters of banana peel act as a metal film interface. This can be described in terms of capacitance in parallel with two charge transfer resistances, as shown in fig $4 \mathrm{c}$. In the present metal-oxide junction kind, the resistance to the moment of electrons across the interface is small compared to the resistance of the moment of cations. Therefore, the capacitance associated with this kind of interface may act as a space charge layer and hence the fast first semicircle occurs at lower frequencies (below $1 \mathrm{k} \mathrm{Hz}$ ) [8].

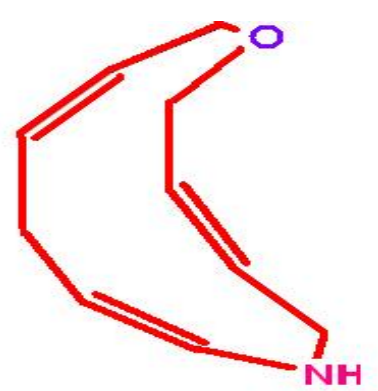

Figure $4 b$

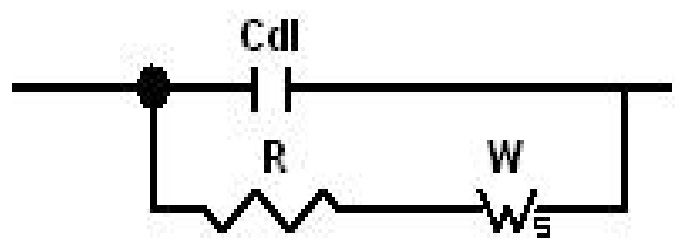

Figure 4c.

Fig $5 \mathrm{a} \& \mathrm{~b}$ shows the room temperature (RT complex impedance plot (Z" vs. Z') of the sample placed in the air as well as in the silicon oil bath. By introducing the additional resistance (Warburg) in the circuit (fig 4c), the data is found to be well fitted and the values are given in the table 1 . The proposed circuit, shown in the fig $4 \mathrm{c}$, seems to be more suitable to the present case on account of its low impedance values and the capacitance (table 1), which is in the order of $10^{-7} \mathrm{~F}$ at lower frequency regime (less than $1 \mathrm{~Hz}$ ). This confirms the blocking of electrode phenomenon.

Robert et al [12] reported the FTIR spectrum of the film formed on metal $(\mathrm{Zn})$ surface after immersing in the sea water containing banana peel-extract. They observed that $\mathrm{C}=\mathrm{C}$ stretching had decreased from $1638 \mathrm{~cm}^{-1}$ to $1626 \mathrm{~cm}^{-1}$ and $\mathrm{O}$ - ring stretching shift from $1051 \mathrm{~cm}^{-1}$ to $1150 \mathrm{~cm}^{-1}$. In addition the NH- stretching had decreased from $3435 \mathrm{~cm}^{-1}$ to $3412 \mathrm{~cm}^{-1}$. Based on this observation, they attributed that nitrogen atom of the $\mathrm{NH}$ - group had coordinated with $\mathrm{Fe}$ formed on the metal surface resulted in the formation of Fe-banana peel extract complex on metal surface. The same result can be corroborated by the complex impedance plot of the sample with $\mathrm{Cu}$-electrodes (obtained in the silicon oil bath) and gold electrodes ( in the air) (fig 5 a \& b). To correlate this mechanism, the complex modulus plot is shown, as an inset figure in fig 5a. From the plot, a sudden change in the modulus value at higher frequency regime clearly indicates the diffusion (Warburg) process. 

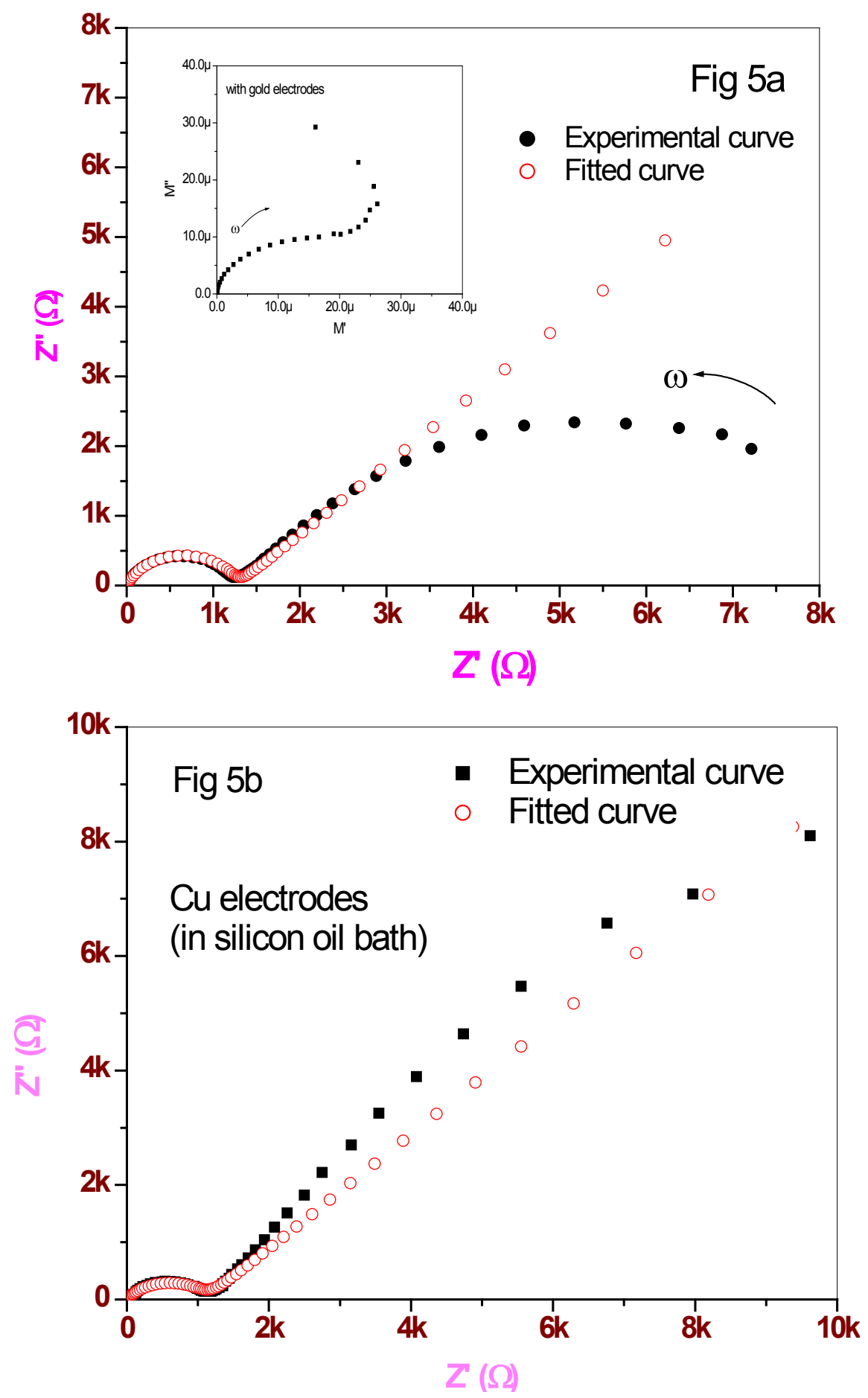

Figure 5. (a) Z" vs. Z' (air with gold electrodes): Inset fig. M" vs. M' (b) Z' vs. Z”' (in silicon oil bath with Cu-electrodes)

In order to confirm the proposed circuit (fig 4c), the real part of admittance (Y') against frequency is shown in the fig 6 . The expression for the real part of the admittance $Y^{\prime}$ (related to conductance) is [13] given below:

$$
\mathrm{Y}^{\prime}=\mathrm{R}^{-1}+\mathrm{A} \omega^{\mathrm{n}} \operatorname{Cos}(\mathrm{n} \pi / 2)
$$

In the spectroscopic plot (fig 6), the regions I, and III indicate the presence of multiple relaxations (non-Debye). In region-II (intermediate regime), $\mathrm{Y}^{\prime}$ is found to be frequency independent nature, where $\mathrm{Y}^{\prime}=\mathrm{R}^{-1}$. The values of $\mathrm{n}$ (slope) come out to be linear gradient, and the term $\log$ [A Cos $(n \pi / 2)]$ can be extracted from the extrapolated data on $\log \mathrm{Y}^{\prime}$ axis (shown as dotted line in fig 6) [13]. The linear dependent nature, in the region-I, is attributed to the diffusion or Warburg phenomenon. To confirm the same, the sample was placed in the silicon oil bath and the extracted data is as shown in fig 6 . In addition, the admittance data obtained at $25{ }^{\circ} \mathrm{C}$ and $10^{\circ} \mathrm{C}$ is also shown in the fig 6 . Based on the results a tentative conclusion is arrived that the blocking electrode phenomenon is predominant with sample-electrode 
interface and therefore the circuit shown in the fig $4 \mathrm{c}$ seems to be more applicable. The typical $\mathrm{n}$ value, obtained from the fitting parameter is found to be more than 0.5. This confirms the high conductive nature of the sample [14]. The admittance (related to conductance) is found to be frequency-dependent nature of the lower frequency regime is considered to be a characteristic feature of the biological system. Keeping the sample in golden electrodes (in air) as well $\mathrm{Cu}$ electrodes (in the silicon oil bath) the obtained admittance data seem to be identical, except at the lower frequency domain (see fig 6). By increasing the frequency, the admittance becomes frequency-independent nature, in the intermediate frequency regime. The results clearly show the significance of onset- blocking process, at lower frequency region. The same thing can be confirmed by comparing the complex-impedance plots shown in fig 5a \& $5 \mathrm{~b}$ respectively. The results are consistent with the literature $[11,12,15-17]$. The impedance values observed in the sample in the log spectroscopic plot (fig 2) is found to be more, when compared to the metal (carbon-steel) immersed in the banana peel extract solution [11]. In addition, hysteresis loops observed in the sample (banana) obtained at lower frequency $(1 \mathrm{~Hz})$ was attributed blocking-regime, and the average time for charging and discharging was found to be different and hence unsymmetrical hysteresis loop was resulted in the $\mathrm{P}$ vs. E measurements. This might lead to paraelectric behaviour [7]. Exhibiting the characteristic peak at intermediate frequency region of the spectroscopic (outside of blocking region) plot (fig 2) is also confirming the paraelectric nature of the banana.

The frequency independent nature of conductance (fig 6) in the intermediate region (below $1 \mathrm{k} \mathrm{Hz}$ ) is mainly due to the transfer of electrons between the two phases. The total conductance is once again attributed to the transport of electrons, holes, anion vacancies and cation vacancies. The moment of anion and cation vacancies within the film under the influence of electric potential gradient and concentration of biological matter can be explained by Fick's law. The total impedance $\left(\mathrm{Z}_{\mathrm{T}}\right)$ can be expressed as:

$$
Z_{\mathrm{T}}=\left(\sigma_{\mathrm{M}} \sigma_{\mathrm{o}} / \sigma_{\mathrm{M}}+\sigma_{\mathrm{o}}\right) \omega^{-1 / 2}(1-\mathrm{j})
$$

$\sigma_{\mathrm{M}}$ and $\sigma_{0}$ are the anion and cation vacancies. The term $Z_{\mathrm{T}}$ is in the form of Warburg impedance for two parallel moving species. However, here the two-limiting cases is: i) moment of cations alone and ii) Moment of anion vacancies alone. Randles plots shown in the figure (7) give more plausible information for low frequency Warburg response anionic and cationic vacancies and its role in the transport mechanism. The plots also show the Warburg coefficient $\left(\sigma=\mathrm{dZ}^{\prime} / \mathrm{dw}^{-\mathrm{n}}\right)$, which is independent of film thickness. The $\sigma$ obtained for $\mathrm{Cu}$-electrode at different temperatures is summarized in table.1

Based on literature values $(2.31 \mathrm{k} \Omega / \mathrm{S})$ it is concluded that oxygen ion vacancies are playing predominant in the conductive mechanism. The interfacial resistance $\left(=\mathrm{R}_{\mathrm{g}} \mathrm{T} / \mathrm{nI}\right.$ o $\mathrm{F}$ where $\mathrm{n}$ is the number if electrons transferred at the interface, $\mathrm{F}$ is Faraday constant and $\mathrm{R}_{\mathrm{g}}$ and $\mathrm{T}$ are ideal gas constant at absolute temperature) can be obtained by fitting the data is also mentioned in the table 1 . The interfacial-charge transfer resistance $(\mathrm{R})$ is in parallel with the double layer capacitance $\left(\mathrm{C}_{\mathrm{dl}}\right)$. This can be corroborated by a complex semicircle appeared in the frequency range of $10-10^{4} \mathrm{~Hz}$ (fig 5). At the lower frequency range below $(1 \mathrm{~Hz})$, a linear response (spike) with angle of $45^{\circ}$ to the real axis also indicates diffusion process.

Table 1. Fitted experimental-Impedance data (based of fig 4c) parameters

\begin{tabular}{|c|c|c|c|c|c|c|}
\hline \multirow{2}{*}{ Sample } & \multirow{2}{*}{$\begin{array}{c}\text { Temperature } \\
\left({ }^{\circ} \mathrm{C}\right)\end{array}$} & $\mathrm{R}(\Omega)$ & $\mathrm{C}_{\mathrm{dl}}(\mathrm{F})$ & $\mathrm{W}(\Omega)$ & $\mathrm{n}$ & \\
\cline { 3 - 6 } & $\mathrm{RT}$ & 1270 & $3.94 \mathrm{E}-07$ & 1450 & 0.74 & $\sigma=\mathrm{dZ}^{\prime} / \mathrm{dw}^{-\mathrm{n}}$ \\
\hline $\begin{array}{c}\text { With gold electrodes } \\
\text { (air medium) }\end{array}$ & $\mathrm{RT}$ & 1125 & $1.73 \mathrm{E}-06$ & 2828 & 0.88 & $\begin{array}{c}\text { At } 25^{\circ} \mathrm{C}-3.7 \mathrm{k} \Omega / \mathrm{S}^{1 / 2} \\
\text { At } 10^{\circ} \mathrm{C}-1.5 \mathrm{k} \Omega / \mathrm{S}^{1 / 2}\end{array}$ \\
\hline $\begin{array}{c}\text { With Cu-electrodes } \\
\text { (silicon oil bath) }\end{array}$ & & & & & $\mathrm{k} \Omega / \mathrm{S}^{1 / 2}$ \\
\end{tabular}




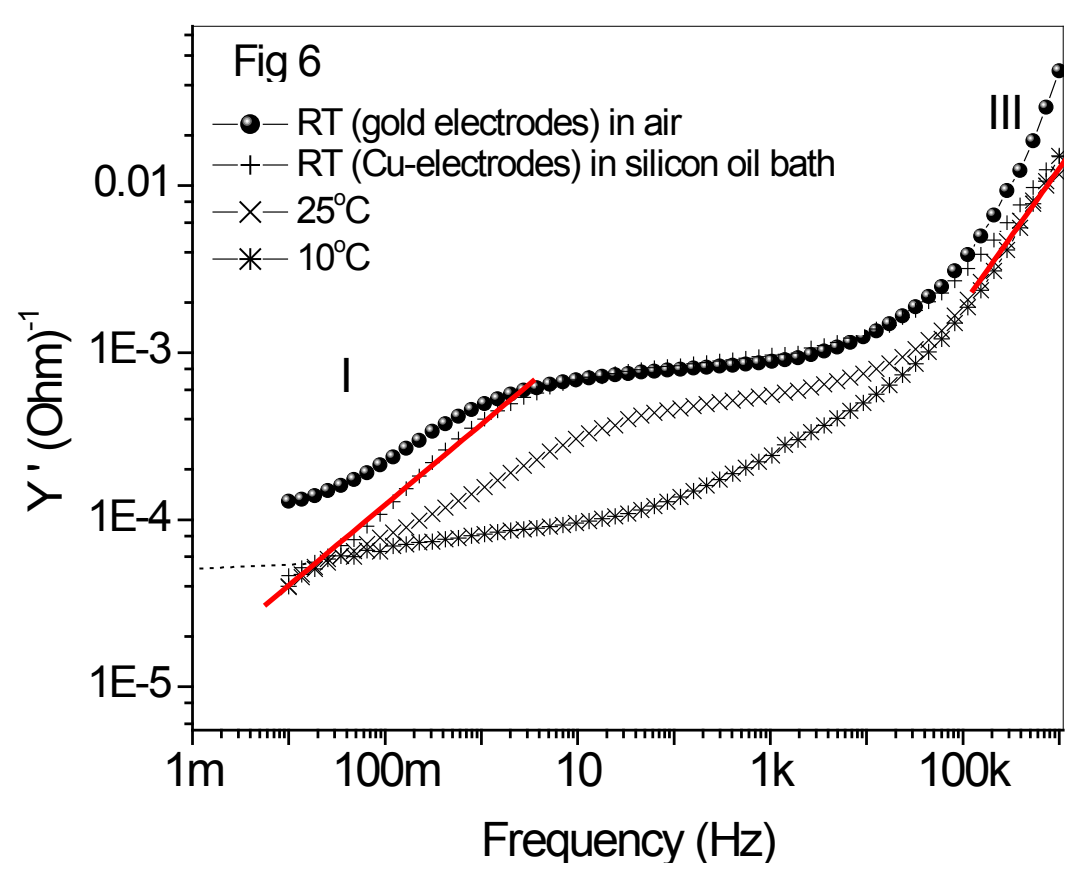

Figure 6. Y' (related to conductance) vs. frequency

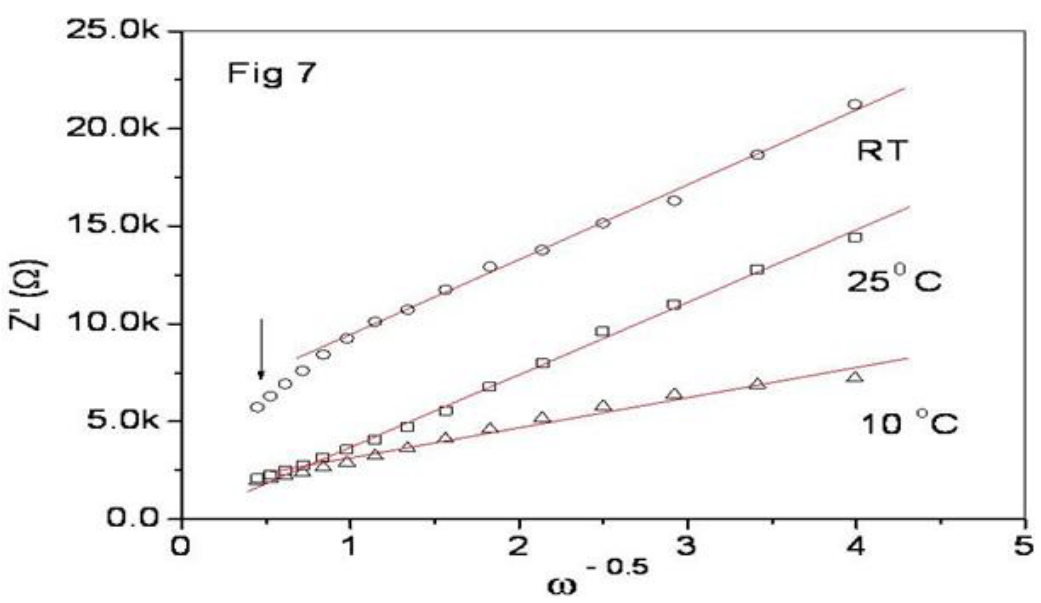

Figure 7. Rundles plot (Z' vs. $\omega^{-0.5}$ )

Based on the above results one can speculate that the lower frequency region or blocking region (below $1 \mathrm{~Hz}$ ) is due to the onset contribution to Warburg-diffusion phenomenon. Jonscher [13] has suggested that the frequency dependent nature of the higher frequency region is attributed to the charge carriers or impurities, and the conduction occurs due to the discontinuous jumps. This kind of screened hopping mechanism is attributed to a cooperative mechanism of the non-Debye type. In the high frequency region (region-III), Universal exponent can be calculated by using the Universal Power law (Jonscher's). Here the value of the gradient (n) is found to be around 0.8. Based on the $\mathrm{n}$-value it is concluded that the increasing the conductivity with frequency is attributed to the flipping of the dipoles due to charge-hopping mechanism between two electrodes and simple interface within the electrode region. This in turn leads to lossy- dielectric nature. This model is dissimilar to the dipolar model, where charges are exchanged between the nearest sites. The overall nature of admittance vs. frequency plot confirms the non-ionic nature and non-intrinsic behaviour of the sample.

\section{Conclusions}

The impedance spectroscopic plot shows two peaks at 1 $\mathrm{Hz}$ and $100 \mathrm{kHz}$. The peaks were well-fitted with the Gaussian relation. By introducing the Warburg resistance, the proposed circuit shown in the fig $4 \mathrm{c}$ seems to be more adequate for the present sample (banana). The spikes observed at lower frequencies (below $1 \mathrm{~Hz}$ ) and Randles plots confirm the presence of blocking elements. In the lower frequency region the response function with respect to the frequency is polarization-dependent and hence it 
influences the charging and discharging process. The results are consistent with complex impedance and admittance plots. At intermediate frequency region, the polarization process is associated with slowly mobile charges of non-ionic nature. Based on the results a tentative conclusion is arrived that banana-peel can act as an inhibitor and protects from metal corrosion.

\section{REFERENCES}

[1] M E Lines and A M Glass, Principles and applications of ferroelectrics and related materials, 1977 (Oxford: Clarendon).

[2] B Jaffe, W R Cook and H Jaffe, Piezoelectric ceramics, 1971 (London Acedamic)

[3] E. Fatuzzo, W.J. Merz, Ferroelectricity 1967 (Amsterdam: London Academic)

[4] J.F. Scott, J. Phys. Conds. Mater 20, 021001 (2008).

[5] T. Yogo, W. Sakamoto, T. Isaji, M. Ichida, A. Nakamura and S. Hirano J. Am. Ceram. Soc., 82, 2672 (1999).

[6] L. Pinulie and M. Alex, App. Phy. Lett., 87, 112903 (2005).

[7] A. Loidl, S. Krohna, J. Hamburger and P. Lunkenheimer, J.
Phy. Cond. Matter 20, 191001 (2008).

[8] J.R. Macdonald, Impedance spectroscopy, 1987 (John Wiley and Sons, New York).

[9] A.K. Jonscher, Dielectric relaxation in solids, 1983 (Chelsa Dielectric Press, London).

[10] W.B. Johnson, N. J. Tolar, G R Miller and I.B. Cutler, J. Phys. Chem. Solids 30, 31 (1969).

[11] M. Sangeetha, S. Rajendran, J. Sathiyabama and P. Prabhaker, Scholar, Research Library, J. Nat. Plant Resour., 2, 601 (2012).

[12] M. Robert Silverstein, Francis X, Web star spectroscopic identification of organic compounds, VI Edition, Wiley student Editor (2007).

[13] A.K. Jonscher, Universal relaxation law, 1996, (Chelsea, Dielectric Press, London)

[14] H.P. Schwan, Introduction mechanism of low-level electromagnetic fields on living systems, Ed., B Norden and C. Ramel, 1992 (Oxford press).

[15] M. Lebrini, F. Robert, C. Cross., Int. J. Electrochem Sci., 5, 1698 (2010).

[16] A. M. Badiea, K.N. Mohan, J. Mat. Eng. And Performance 18,1264 (2009).

[17] A. Sharmile, A.A. Prema, P.A. Sahayaraj, Rasayana J of Chem., 3, 74 (2010). 